Article

\title{
An AMOLED Pixel Circuit Based on LTPS Thin-film Transistors with Mono-Type Scanning Driving
}

\author{
Jianyuan Ke, Lianwen Deng *, Liying Zhen, Qing Wu, Congwei Liao, Heng Luo \\ Shengxiang Huang \\ School of Physics and Electronics, Central South University, Changsha 410083, China; \\ ke_jianyuan@csu.edu.cn (J.K.); liyingzhen@csu.edu.cn (L.Z.); wuwuqing@csu.edu.cn (Q.W.); \\ liaocw@csu.edu.cn (C.L.); luohengcsu@csu.edu.cn (H.L.); hsx351@csu.edu.cn (S.H.) \\ * Correspondence: denglw@csu.edu.cn; Tel.: +86-13787206916
}

Received: 12 March 2020; Accepted: 26 March 2020; Published: 28 March 2020

check for updates

\begin{abstract}
Using low-temperature poly-silicon thin-film transistors (LTPS TFTs) as a basis, a pixel circuit for an active matrix organic light-emitting diode (AMOLED) with narrow bezel displays was developed. The pixel circuit features mono-type scanning signals, elimination of static power lines, and pixel-integrated emitting control functions. Therefore, gate driver circuits of the display bezel can be simplified efficiently. In addition, the pixel circuit has a high-resolution design due to an increase of the pulse width of the scan signal to extend the threshold voltage and internal-resistance drop (IR drop) detection period. Further, regarding the influences of process-voltage-temperature (PVT) variation in the pixel circuit, comparison investigations were carried out with the proposed circuit and other pixel circuits with mono-type scanning signals using Monte Carlo analysis. The feasibility of the proposed pixel circuit is well demonstrated, as the current variations can be reduced to $2.1 \%$ for the supplied power reduced from $5 \mathrm{~V}$ to $3 \mathrm{~V}$ due to IR drop, and the current variation is as low as $10.6 \%$ with operating temperatures from -40 degrees to 85 degrees.
\end{abstract}

Keywords: AMOLED; LTPS TFT; pixel circuit; IR drop; Monte Carlo

\section{Introduction}

Active-matrix organic light-emitting diode (AMOLED) displays have an important presence in mainstream next-generation displays due their extremely high contrast ratio, fast electrical-optical response, and excellent compatibility with flexible electronics [1,2]. Although different AMOLED backplane solutions have been discussed over the past decades [3,4], for state-of-the-art AMOLED displays, low-temperature poly-silicon thin-film transistors (LTPS TFTs) show better performance. Compared with other types of TFTs, LTPS TFTs exhibit higher mobility and better stability in terms of electrical characteristics [5,6]. However, the drawbacks of LTPS TFTs lie in the obvious variations in the threshold voltage $\left(V_{T H}\right)$ and mobility $[7,8]$, which hinder the implementation of high-quality AMOLEDs with uniform display for larger dimensions. For OLED pixel circuit design, the method of detecting and compensating the non-uniformities in TFT characteristics is essential. In addition, the internal-resistance drop (IR drop) on the power line brings additional variation to OLED displays in larger-sized AMOLED panels $[9,10]$. Further, for co-design of pixel circuits with TFT-integrated gate drivers, it is necessary to meet the requirements of ultra-narrow border displays.

AMOLED pixel schematics can be categorized into three types: voltage programming methods [11,12], current programming methods [13,14], and external compensation methods [15,16]. Voltage-programming methods are mainstream for LTPS OLEDs because current programming methods need long settling times for small currents, and external compensation methods are too complicated. However, for voltage programming methods, the pixel circuit is required to correct 
variations in $V_{T H}$, mobility, and $V_{D D}$. Thus, Lin et al. proposed a feedback pixel circuit to calibrate the gate voltage distortion of driving TFTs during the light emission time [17]. Lee et al. re-used the scan line for supplying power to replace the conventional power line to increase the aperture ratio [18]. Further, to avoid image flicker, Lin et al. employed overlapping compensation waveforms to extend the $V_{T H}$ detection time and to suppress the OLED current during the compensation time [19]. These previous investigations illustrate that, through a proper circuit topology and timing diagram to offset $V_{T H}$ variations and IR drop, a good display uniformity can be obtained. However, for conventional schematics, the pixel circuit array requires multiple scan signals with different types, which complicates both the pixel circuit and the external gate driver circuit.

In this paper, a compensation AMOLED pixel circuit with mono-type scanning lines is proposed. An emit-controlling function is integrated within the pixel circuit, and the conventional $V_{D D}$ line is also eliminated. Table 1 shows the comparison among the proposed and previous pixel circuits. For conventional pixel circuits, complicated gate drivers are required, as multiple types of scan signals are used, while the proposed circuit has fewer control signals for simpler external drivers. Comparison investigations are carried out with the proposed circuit and other AMOLED pixel circuits with mono-type scanning lines.

Table 1. Comparison among the proposed and previous pixel circuits. TFT: thin-film transistor.

\begin{tabular}{|c|c|c|c|c|c|c|}
\hline Reference & $\begin{array}{c}\text { TFT } \\
\text { Number }\end{array}$ & TFT Type & $\begin{array}{l}\text { Number of } \\
\text { Scan Lines }\end{array}$ & $\begin{array}{c}\text { Scanning } \\
\text { Signal Types }\end{array}$ & $\begin{array}{c}\text { Flicker } \\
\text { Prevention }\end{array}$ & $\begin{array}{l}\text { Detection Time } \\
\text { Extension }\end{array}$ \\
\hline [5] & $6 \mathrm{~T} 1 \mathrm{C}$ & $\begin{array}{l}\text { p-type, } \\
\text { poly-Si }\end{array}$ & 3 & 3 & No & Yes \\
\hline [7] & $5 \mathrm{~T} 2 \mathrm{C}$ & $\begin{array}{l}\text { p-type, } \\
\text { poly-Si }\end{array}$ & 3 & 3 & Yes & Yes \\
\hline [8] & $5 \mathrm{~T} 2 \mathrm{C}$ & $\begin{array}{l}\text { p-type, } \\
\text { poly-Si }\end{array}$ & 4 & 4 & Yes & Yes \\
\hline [9] & $5 \mathrm{~T} 2 \mathrm{C}$ & $\begin{array}{l}\text { n-type, } \\
\text { a-Si:H }\end{array}$ & 3 & 3 & Yes & Yes \\
\hline [11] & $3 \mathrm{~T} 2 \mathrm{C}$ & $\begin{array}{l}\text { p-type, } \\
\text { poly-Si }\end{array}$ & 2 & 2 & No & No \\
\hline [17] & $5 \mathrm{~T} 2 \mathrm{C}$ & $\begin{array}{l}\text { p-type, } \\
\text { poly-Si }\end{array}$ & 3 & 3 & Yes & Yes \\
\hline This work & $7 \mathrm{~T} 2 \mathrm{C}$ & $\begin{array}{l}\text { p-type, } \\
\text { poly-Si }\end{array}$ & 2 & 1 & Yes & Yes \\
\hline
\end{tabular}

\section{Pixel Circuit Description}

Figure 1 shows the schematic and timing diagram of proposed pixel circuit, which consists of seven TFTs and the two capacitors $\left(C_{S T}\right.$ and $\left.C_{T H}\right)$. The specifications for the proposed pixel circuit are shown in Table 2. Here, P1 is the driving transistor, and P2 to P7 are switching transistors. The voltage across $C_{T H}$ is reset by the switching transistors P2, P4, P5, and P7 during the initialization phase. The $V_{T H}$ of $\mathrm{P} 1$ and supplied power information are stored by the right terminal of $C_{T H}$ by the turning on of P2 for the detection phase. The data signal is transferred to the left terminal of $C_{T H}$ through P3, and the OLED starts to emit following the negative pulse of $\mathrm{V}_{\mathrm{SCAN}}(n+2)$ through the pull-downing of P6. Figure 2 shows the layouts of a single subpixel of the proposed pixel circuit and 5T2C bootstrap pixel circuit [20]. Although the proposed pixel circuit uses more TFTs than 5T2C, the layout area has not increased much, so it has little effect on resolution. The working process of the pixel circuit will be described in detail. 


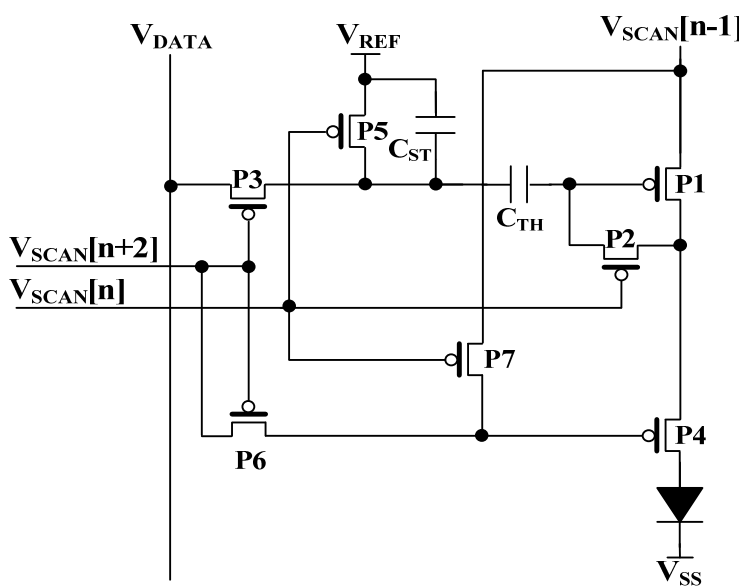

(a)

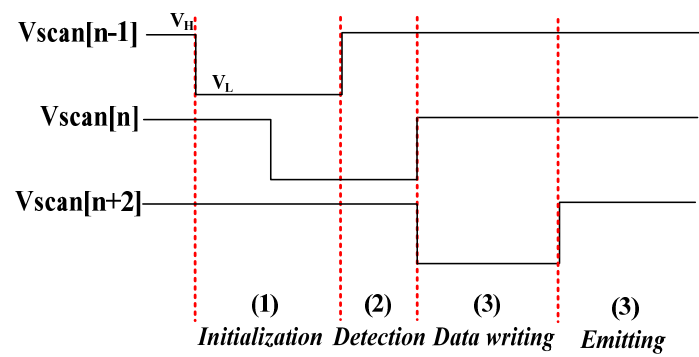

(b)

Figure 1. The proposed active matrix organic light-emitting diode (AMOLED) pixel circuit. (a) The circuit schematic, and (b) the timing diagram. $V_{T H}$ : threshold voltage; $V_{D A T A}$ : data voltage.

Table 2. Parameters of the proposed pixel circuit.

\begin{tabular}{cccc}
\hline Parameter & Value & Parameter & Value \\
\hline$W / L(P 1)$ & $4 \mu \mathrm{m} / 10 \mu \mathrm{m}$ & $W / L(P 7)$ & $4 \mu \mathrm{m} / 4 \mu \mathrm{m}$ \\
$W / L(\mathrm{P} 2)$ & $4 \mu \mathrm{m} / 4 \mu \mathrm{m}$ & $V_{S C A}$ & $-10-5 \mathrm{~V}$ \\
$W / L(P 3)$ & $4 \mu \mathrm{m} / 4 \mu \mathrm{m}$ & $V_{S S}$ & $-4 \mathrm{~V}$ \\
$W / L(\mathrm{P} 4)$ & $4 \mu \mathrm{m} / 4 \mu \mathrm{m}$ & $V_{R E F}$ & $-1 \mathrm{~V}$ \\
$W / L(P 5)$ & $4 \mu \mathrm{m} / 4 \mu \mathrm{m}$ & $C_{T H}$ & $0.1 \mathrm{pF}$ \\
W/L (P6) & $4 \mu \mathrm{m} / 4 \mu \mathrm{m}$ & $C_{S T}$ & $0.3 \mathrm{pF}$ \\
\hline
\end{tabular}

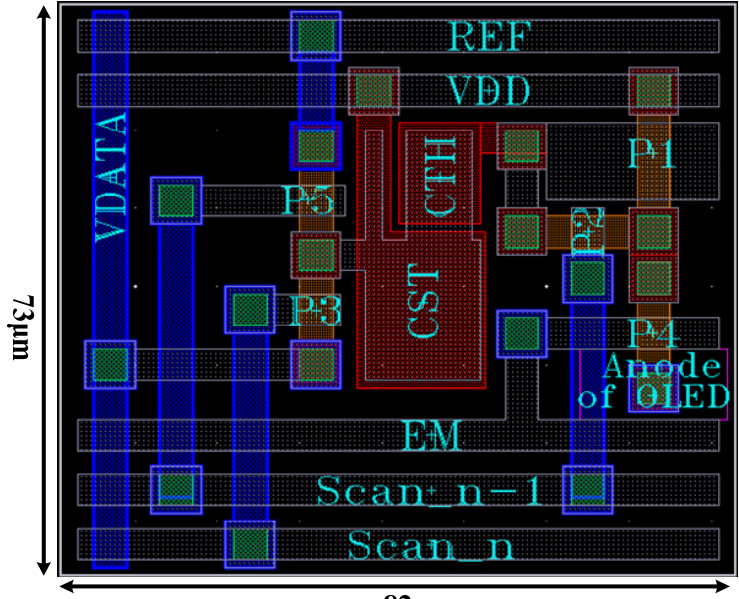

$82 \mu \mathrm{m}$

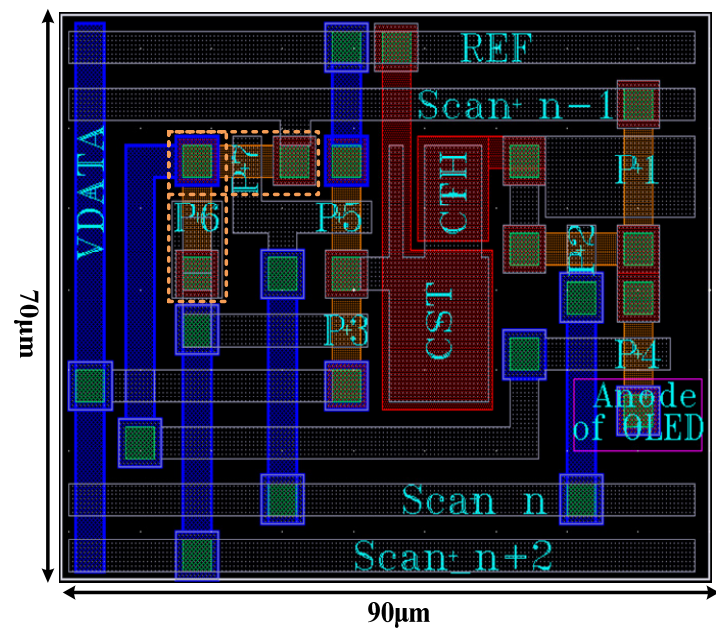

(b)

Figure 2. Layout of the pixel circuit. (a) The 5T2C bootstrap pixel circuit, and (b) the proposed AMOLED pixel circuit.

\subsection{Initialization Phase}

During the initialization phase, P2, P5, and P7 are turned on as both $\mathrm{V}_{\mathrm{SCAN}}(n-1)$ and $\mathrm{V}_{\text {SCAN }}(n)$ are at a low level. Then the gate voltage of $\mathrm{P} 4$ is pulled down, and $\mathrm{P} 4$ is turned on for resetting the drain electrode of P1 with a relatively low voltage level, i.e., below the turning-on voltage of OLED $\left(V_{O L E D}\right)$. In addition, the gate and the drain electrodes of the driving transistor P1 are shorted by P2 and the gate voltage of P1 is also discharged through P4, while the left electrode of $C_{T H}$ is initialized with $V_{R E F}$ by P5. The electrical hysteresis phenomenon in P-type LTPS transistors should be considered, 
i.e., the drain-to-source current varies with different gate-sweeping directions, which relates with short-term image sticking issues [21,22]. As $C_{T H}$ is properly initialized before the programming phases, the hysteresis effects can be efficiently suppressed.

\subsection{Detection Phase}

During the detection phase, $\mathrm{P} 4$ is turned off as $\mathrm{V}_{\mathrm{SCAN}}(n-1)$ switches to a high level. Then, the gate and the drain electrodes of $\mathrm{P} 1$ are gradually charged until the voltage difference between the gate and the source of P1 is $V_{T H}$. By the end of the detection phase, the gate voltage of P1 approximates the voltage of the power line minus $\left|V_{T H}\right|$. Since P1 operates in the saturation region, the transient response for the gate voltage of $\mathrm{P} 1$ can be expressed as:

$$
\left(C_{T H}+C_{P 1 \_G S}+C_{P 2 \_G D}\right) \frac{d V_{G}(t)}{d t}=\frac{1}{2} \mu C_{O X} \frac{W}{L}\left[V_{H}-V_{G}(t)-\left|V_{T H}\right|\right]^{2},
$$

where $C_{P 1 \_G S}, C_{P 2} G D, \mu, C_{O X}, W / L$, and $V_{H}$ are the gate-to-source capacitance of P1, the gate-to-drain capacitance of $\mathrm{P} 2$, field-effective mobility, gate capacitance per unit area, ratio of the channel width to channel length of P1, and the high level of the scan signal, respectively. Assuming the initial value of the P2's gate electrode as $V_{O}, V_{G}$ can be solved as:

$$
V_{G}(t)=V_{H}-\left|V_{T H}\right|-\frac{1}{\left.\frac{\mu C_{O X} W\left(t-t_{0}\right)}{2 L\left(C_{T H}+C_{P 1} G S+C_{P 2} G D\right.}\right)}+\frac{1}{V_{O}-V_{H}+\left|V_{T H}\right|},
$$

where $t_{0}$ and $t$ are the start and end times of the charging process, respectively. The compensating efficiency might be reversely affected by the detection accuracy of $V_{T H}$ and $V_{H}$. As shown in Equation (2), for accurate detection of $V_{T H}$ and $V_{H}$, a relatively long charging time is required. However, due to the increasing of display resolution, it becomes difficult to extend the detection time.

\subsection{Data Writing Phase}

During the data writing phase, P3 and P6 are turned on as the levels of Vscan $(n-1)$ and Vscan $(n)$ are low, and P2, P5, and P7 are turned off as the level of Vscan $(n+2)$ is high. Then the data voltage, i.e., $V_{D A T A}$, is transferred to the left electrode of $C_{T H}$ through P3. According to the charge conservation law, the voltage at the right electrode of $C_{T H}$ can be expressed as:

$$
V_{G}^{\prime}=V_{G}-\frac{C_{P 1 \_G D} V_{G}}{C_{T H}+C_{P 1 \_G S}+C_{P 1 \_G D}+C_{P 2 \_G D}}+\frac{C_{T H}\left(V_{D A T A}-V_{R E F}\right)}{C_{T H}+C_{P 1 \_G S}+C_{P 1 \_G D}+C_{P 2 \_G D}},
$$

Considering that $C_{P 1_{-} G D}, C_{P 1_{-} G D}$, and $C_{P 2_{-} G D}$ are much smaller than $C_{T H}$, one can simplify Equation (3) to:

$$
V_{G}^{\prime}=V_{G}+V_{D A T A}-V_{R E F},
$$

\subsection{Emitting Phase}

Finally, in the emitting phase, the driving TFT provides the current to OLED pixel, which can be expressed by:

$$
\begin{aligned}
& I_{O L E D}=\frac{1}{2} \mu C_{O X} \frac{W}{L}\left(V_{H}-V_{G}^{\prime}-\left|V_{T H}\right|\right)^{2} \\
& =\frac{1}{2} \mu C_{O X} \frac{W}{L}\left(V_{H}-V_{H}+\left|V_{T H}\right|+\frac{1}{\frac{\mu C_{O X} W\left(t-t_{0}\right)}{2 L\left(C_{T H}+C_{P 1_{-} G S}+C_{P P_{-} G D}\right)}+\frac{1}{V_{O}-V_{H}+\left|V_{T H}\right|}}+V_{R E F}-V_{D A T A}-\left|V_{T H}\right|\right)^{2} \\
& =\frac{1}{2} \mu C_{O X} \frac{W}{L}\left(V_{R E F}-V_{D A T A}+\frac{1}{\frac{\mu C_{O X} W\left(t-t_{0}\right)}{2 L\left(C_{T H}+C_{P 1} G S+C_{P 2} G D\right)}+\frac{1}{V_{O}-V_{H}+\mid V_{T H}}}\right)^{2}
\end{aligned}
$$


Therefore, $I_{O L E D}$ is determined by $V_{R E F}$ and $V_{D A T A}$, being almost independent of $V_{T H}$ and $V_{H}$. As shown in Equation (5), the third term in the parenthesis is reversely related with the effective mobility, and then the pixel circuit also renders certain compensation function of mobility non-uniformities. In addition, $\mathrm{P} 4$ is maintained off for the initialization and detection phases, the leakage current of the OLED can be suppressed efficiently, and improved contrast ratio with reduced flicker can be obtained.

\section{Gate Driver Description}

For the co-design of pixel circuit and gate driver circuit, the Rensselaer Polytechnic Institute (RPI) model with Level 36 is used to reproduce the electrical performance of the p-type LTPS TFTs. Figure 3 demonstrates the comparison of the measured and simulated transfer characteristics of LTPS TFT in a logarithm scale. Calculated using the classic current-voltage model of MOSFET, the field-effect mobility, the threshold voltage and the sub-threshold swing for the LTPS TFT are approximately $72 \mathrm{~cm}^{2}(\mathrm{~V} \cdot \mathrm{s})^{-1}$, $-1.1 \mathrm{~V}$, and $0.74 \mathrm{~V} /$ decade, respectively.

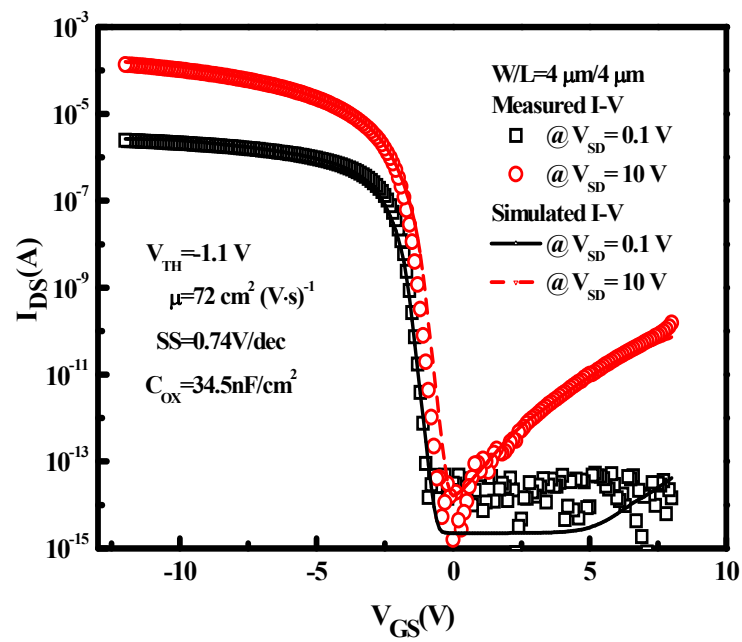

Figure 3. Measured and simulated transfer characteristics of the low-temperature poly-silicon thin-film transistor (LTPS TFT) with an aspect ratio of $4 \mu \mathrm{m} / 4 \mu \mathrm{m}$. $C_{O X}$ : gate capacitance per unit area; $\mu$ : field-effective mobility; $S S$ : sub-threshold swing.

For the proposed pixel circuit, the high-to-low pulses with line-by-line scanning sequences are needed. Figure 4 shows the gate driver unit, which consists of six TFTs and one capacitor. The parameters of the gate driver unit are shown in Table 3 and the operating mechanism has been detailed elsewhere [23]. In order to generate the required scan signal, four clocks with overlapped waveforms are used. Figure 5 shows the block diagram of the gate driver with detection time half of the scan pulse. The simulation results are illustrated in Figure 6. The devised gate driver circuit is well verified, and a rail-to-rail output range, i.e., from $-10 \mathrm{~V}$ to $5 \mathrm{~V}$, can be obtained for the gate driver, with the rising and falling times of $1.68 \mu \mathrm{s}$ and $1.76 \mu$ s for loading resistance of $2 \mathrm{~K} \Omega$ and loading capacitance of $200 \mathrm{pF}$.

Table 3. Parameters of the gate driver circuit.

\begin{tabular}{cccc}
\hline Parameter & Value & Parameter & Value \\
\hline$W / L(G 1)$ & $500 \mu \mathrm{m} / 4 \mu \mathrm{m}$ & $W / L(G 6)$ & $10 \mu \mathrm{m} / 4 \mu \mathrm{m}$ \\
$W / L(G 2)$ & $30 \mu \mathrm{m} / 4 \mu \mathrm{m}$ & $C_{1}$ & $0.8 \mathrm{pF}$ \\
$W / L(G 3)$ & $20 \mu \mathrm{m} / 4 \mu \mathrm{m}$ & $C K$ & $-10-5 \mathrm{~V}$ \\
$W / L(G 4)$ & $10 \mu \mathrm{m} / 4 \mu \mathrm{m}$ & $I N$ & $-10-5 \mathrm{~V}$ \\
$W / L(G 5)$ & $10 \mu \mathrm{m} / 4 \mu \mathrm{m}$ & $V_{G H}$ & $5 \mathrm{~V}$ \\
\hline
\end{tabular}




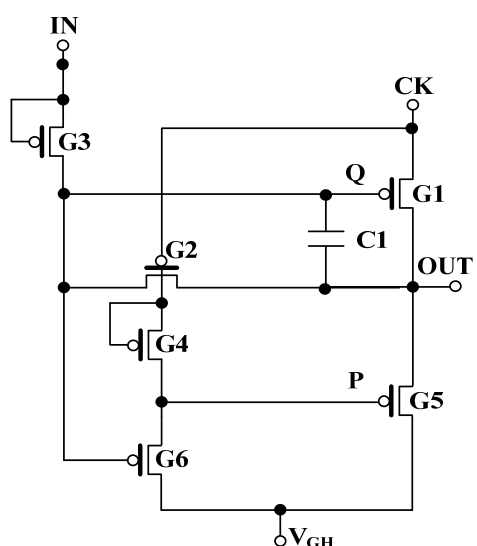

(a)

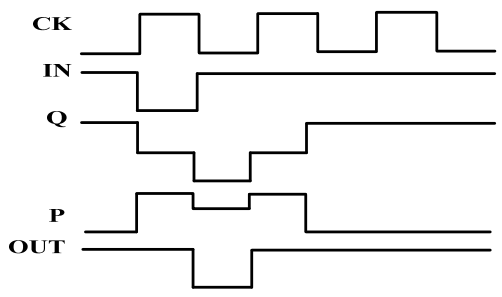

(b)

Figure 4. The integrated gate driver unit, with (a) the schematic, and (b) the timing diagram.

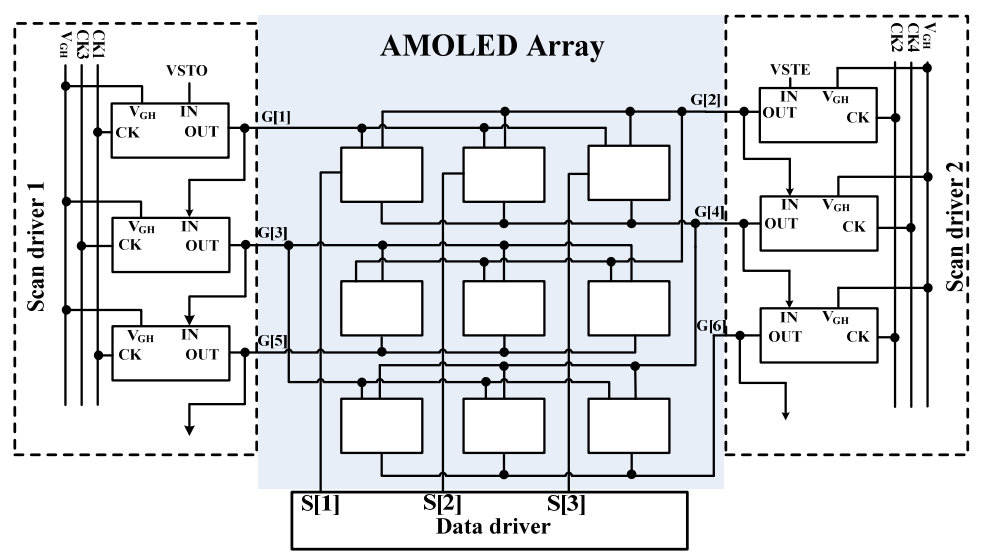

Figure 5. The block diagram of the proposed gate driver circuit.

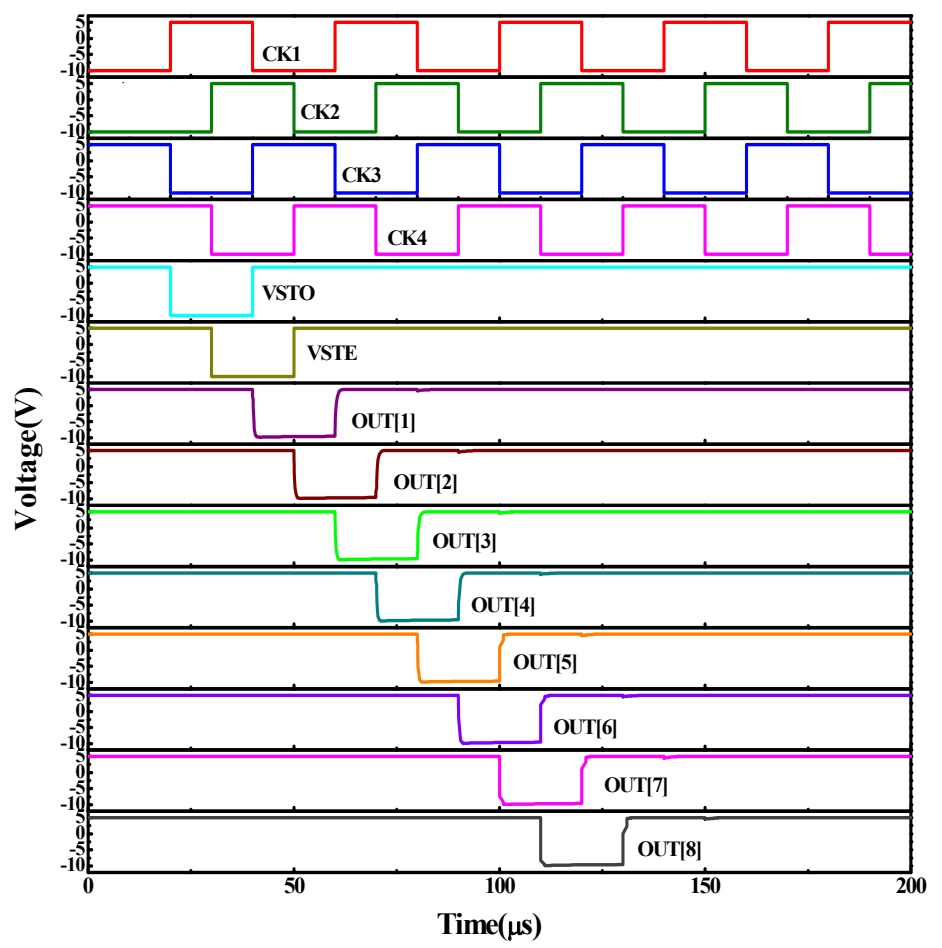

Figure 6. The transient response of the proposed gate driver circuit with $R_{L}=2 \mathrm{~K} \Omega$ and $C_{L}=200 \mathrm{pF}$. 


\section{Results and Discussion}

As mentioned above, a sufficient detection time for $V_{T H}$ and $V_{H}$ is required to improve the compensation accuracy of pixel circuit. However, with the increase of display resolution and frame rate, the detection time for $V_{T H}$ and $V_{H}$ is decreasing. To be more specific, for frame rate of $60 \mathrm{~Hz}$, the row times for the resolution of full-high-definition (FHD) (1920 red-green-blue(RGB) $\times 1080)$, quad-high definition (QHD) (2560 RGB $\times 1440)$, and ultra-high-definition (UHD) (3840 RGB $\times 2160)$, are $15.4 \mu \mathrm{s}$, $11.5 \mu \mathrm{s}$, and $7.7 \mu \mathrm{s}$, respectively. In the proposed circuit, the detection time can be increased by adjusting of the scanning signals. In addition to increase the scan pulse width, modifications of the scanning sequence for the pixel circuit include, replacing the original $\mathrm{V}_{\mathrm{SCAN}}(n-1)$ and $\mathrm{V}_{\mathrm{SCAN}}(n+2)$ with $\mathrm{V}_{\text {SCAN }}(n-2)$ and $\mathrm{V}_{\text {SCAN }}(n+3)$, respectively. Then, the detection time is increased from $2 \mathrm{H}$ to $3 \mathrm{H}$, where $1 \mathrm{H}$ presents the one horizontal time.

Figure 7 shows the comparison of proposed circuit current error rate for different resolutions in conventional driving method (detection time $=1 \mathrm{H}$ ) and detection time adjustable driving method (detection time $=2 \mathrm{H}$ ). For the conventional driving method, i.e., scan width $=2 \mathrm{H}$, and detection time $=1 \mathrm{H}$, the current error rate increases for higher resolution. For improved driving method, i.e., scan width $=3 \mathrm{H}$, and detection time $=2 \mathrm{H}$, the current error rate is almost independent of the display resolution.

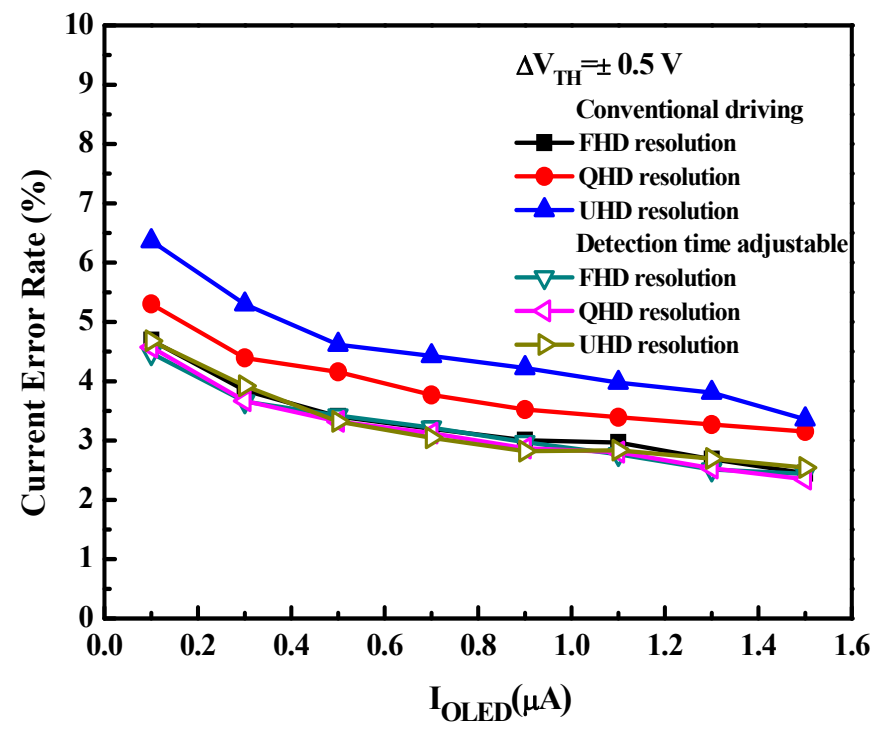

Figure 7. Current error rate versus $\mathrm{I}_{\mathrm{OLED}}$ for different driving schemes with various resolution formats. FHD: full-high-definition; QHD: quad-high definition; UHD: ultra-high-definition.

In addition to the length of the detection period, the actual performance of pixel circuit depends on process-voltage-temperature (PVT) factors [24,25], namely the electrical characteristic variations of poly-silicon TFT due to the fabrication process, fluctuations in the power supply, and the distribution of ambient temperature. Due to PVT effects, current-to-voltage curve will shift from the expected one. In the following, PVT influences on the pixel circuit performance with the resolution of 1920 RGB $\times 1080$ is investigated. Then, PVT performances of the proposed 7T2C circuit schematic are compared with that of the conventional 2T1C pixel circuit and a 4T1C pixel circuit, which compensates $V_{T H}$ and mobility of the driving TFT through discharging of a mirrored TFT. Figure 8 shows the circuit and timing diagram. The merit of the 4T1C circuit schematic is that the structure is relatively simple, and the required scan and data drive waveforms are as simple as the $2 \mathrm{~T} 1 \mathrm{C}$ scheme. 


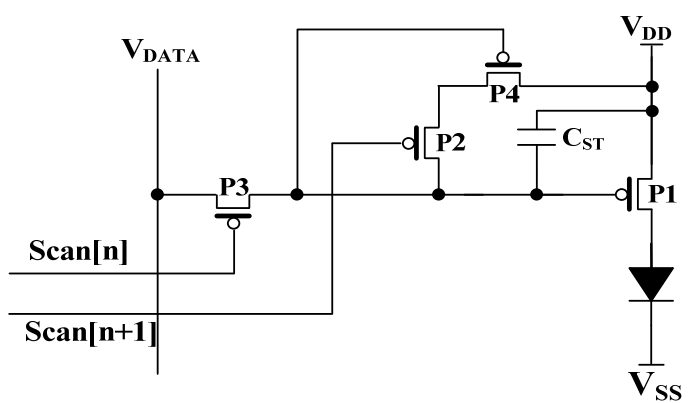

(a)

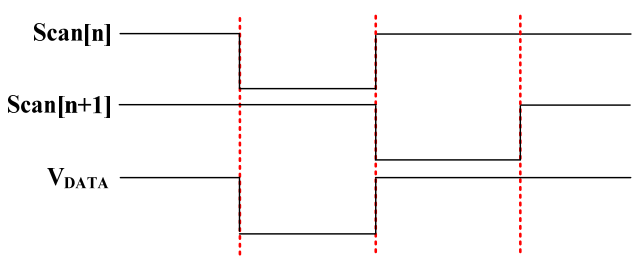

(b)

Figure 8. The mirror-discharge based AMOLED pixel circuit, with (a) the pixel schematic, and (b) the timing diagram.

Due to the grain boundary and silicon orientation variations, the current-to-voltage characteristics of the poly-Si TFT tend to vary from pixel to pixel. Here, Gaussian statistical distribution is used to model the threshold-voltage of the driving TFT. Monte Carlo simulations were performed for threshold voltage variations of $20 \%$ from the nominal values for TFT of 7T2C, 2T1C, and 4T1C pixel circuits and Figure 9 shows Monte Carlo simulation results of the output current error with the sample number of 1000. The mean value of pixel current is $1 \mu \mathrm{A}$, and the standard deviations for the pixel current are $4.6 \mathrm{nA}, 8.5 \mathrm{nA}$, and $151.6 \mathrm{nA}$ for the 7T2C, 4T1C, and 4T1C pixel schematics, respectively. These results further confirm that the proposed pixel circuit has higher luminance uniformity and a stronger immunity to process variations than the conventional $2 \mathrm{~T} 1 \mathrm{C}$ pixel circuit and the $4 \mathrm{~T} 1 \mathrm{C}$ pixel circuit.

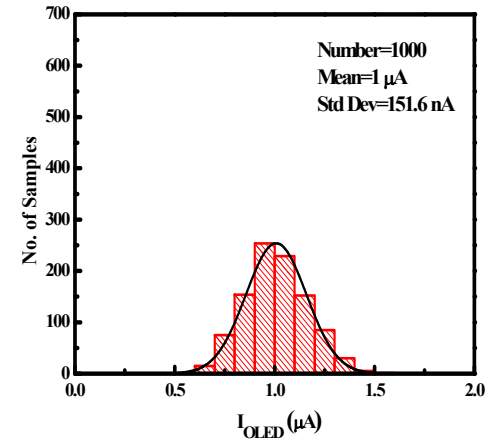

(a)

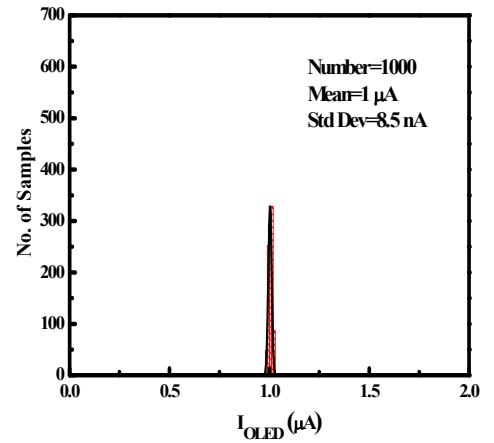

(b)

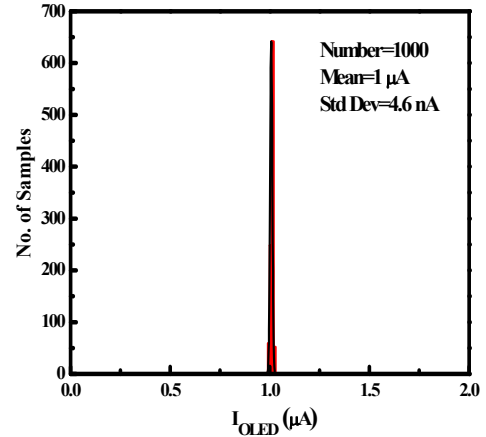

(c)

Figure 9. Monte Carlo analysis of the emission current with $V_{T H}$ in a Gaussian statistical distribution when the relative error is $20 \%$. (a) The conventional 2T1C pixel circuit, (b) the 4T1C pixel circuit, and (c) the proposed pixel circuit.

In addition, due to the metal line resistance, voltage drop along the power supply line is inevitable. For larger display panel, the IR drop effect is further deteriorated. Figure 10 shows the current error rate versus IR drop with three pixel circuits. For the conventional 2T1C pixel, abrupt current error rate is caused by IR drop. In the case $V_{H}$ drops from $5 \mathrm{~V}$ to $3 \mathrm{~V}$, the current error is reduced to $16.7 \%$ and $2.1 \%$ for the $4 \mathrm{~T} 1 \mathrm{C}$ pixel circuit and the 7T2C schematic, respectively. Thus, the proposed pixel circuit shows stronger immunity to the IR drop issue than other pixel circuits, which benefits the implementation of a large-dimension AMOLED panel. For actual products, the operating temperature for AMOLED panels is in the range of -40 degrees to 85 degrees. Figure 11 shows the OLED current versus different temperature for three pixel circuits. The current magnitude is increased by $32.1 \%$ and $25.7 \%$ with the temperature increase from -40 degrees to 85 degrees for $2 \mathrm{~T} 1 \mathrm{C}$ and $4 \mathrm{~T} 1 \mathrm{C}$ circuit. The proposed pixel circuit has much reduced temperature dependence, as the current error is reduced to $10.6 \%$ for the temperature variations. 


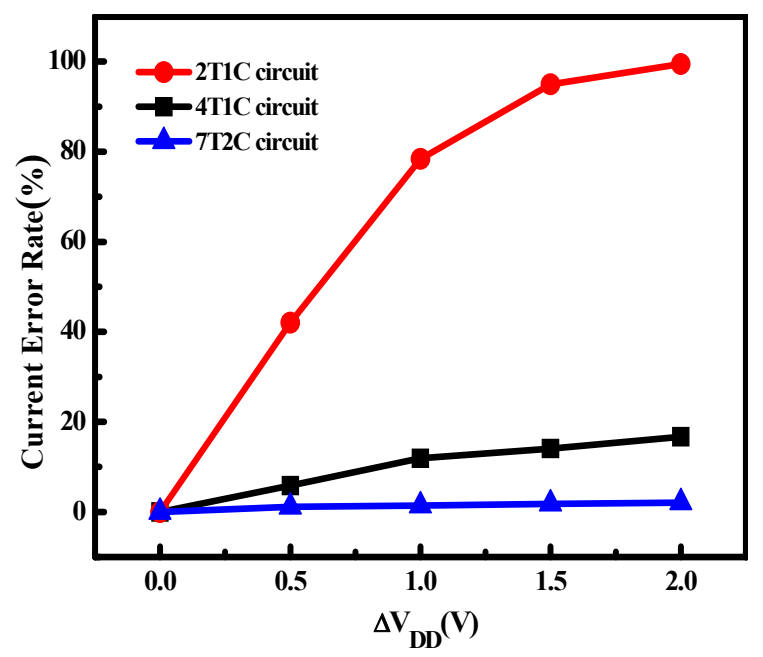

Figure 10. Evolution of $I_{O L E D}$ error rate versus increased IR drop.

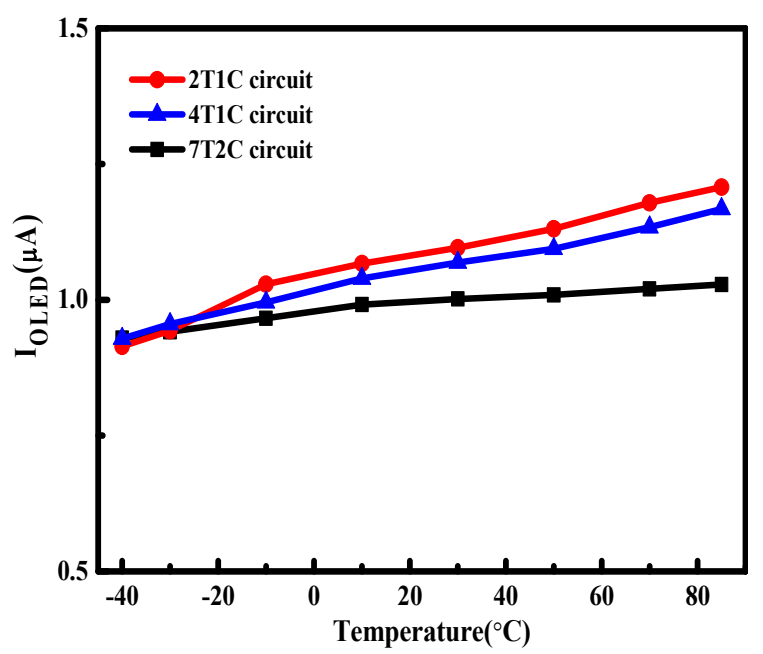

Figure 11. Evolution of $I_{O L E D}$ versus temperature.

\section{Conclusions}

This paper proposed a pixel circuit suitable for a narrow border display, which can compensate for threshold voltage non-uniformity and IR drop, and increase the detection time by increasing the signal width at high resolution. The pixel circuit requires mono-type scanning signals, which benefits simplification of the external driving circuit for narrow border displays. The conventional $V_{D D}$ line is replaced by the scanning line to suppress the leakage current for the initial stage. The proposed pixel circuit has a good compensation effect and stability for temperature variations, the simulation results show that the current variation is reduced to $2.1 \%$ for the supplied power reduced from $5 \mathrm{~V}$ to $3 \mathrm{~V}$, and the current variation is as low as $10.6 \%$ for temperatures from -40 degrees to 85 degrees.

Author Contributions: Conceptualization, J.K. and C.L.; methodology, J.K.; software, J.K. and C.L.; validation, J.K.; formal analysis, C.L. and S.H.; investigation, H.L. and Q.W.; writing-original draft preparation, J.K.; writing-review and editing, J.K., C.L., and L.Z.; supervision, L.D. and C.L.; funding acquisition, L.D. All authors have read and agreed to the published version of the manuscript.

Funding: This work was supported in part by the National Key Research and Development Program of China under Grant 2017YFA0204600, and by the Fundamental Research Funds for the Central Universities of Central South University (No. 2019zzts419).

Conflicts of Interest: The authors declare no conflict of interest. 


\section{References}

1. Park, C.I.; Seong, M.; Kim, M.A.; Kim, D.; Jung, H.; Cho, M.; Lee, S.H.; Lee, H.; Min, S.; Kim, J.; et al. 54-1: Distinguished Paper: World 1st Large Size 77-inch Transparent Flexible OLED Display. SID Symp. Dig. Tech. Pap. 2018, 49, 710-713. [CrossRef]

2. Seol, H.-C.; Ra, J.-H.; Hong, S.-K.; Kwon, O.-K. An AMOLED Panel Test System Using Universal Data Driver ICs for Various Pixel Structures. IEEE Trans. Electron. Devices 2016, 64, 189-194. [CrossRef]

3. Qin, T.; Liao, C.; Huang, S.; Yu, T.; Deng, L. Analytical drain current model for symmetric dual-gate amorphous indium gallium zinc oxide thin-film transistors. Jpn. J. Appl. Phys. 2017, 57, 14301. [CrossRef]

4. Li, C.; Liao, C.-W.; Yu, T.-B.; Ke, J.-Y.; Huang, S.; Deng, L. Concise Modeling of Amorphous Dual-Gate In-Ga-Zn-O Thin-Film Transistors for Integrated Circuit Designs. Chin. Phys. Lett. 2018, 35, 027302. [CrossRef]

5. Lin, C.L.; Chen, P.S.; Chen, H.M.; Chen, F.H. A novel p-Type LTPS TFT pixel circuit compensating for threshold voltage and mobility variations. J. Disp. Tech. 2014, 10, 995-1000.

6. Wadhwa, N.; Bahubalindruni, P.G.; Chapagai, K.; Goes, J.; Deb, S.; Barquinha, P. Sixth-order differential Sallen-and-Key switched capacitor LPF using a-IGZO TFTs. Int. J. Circuit Theory Appl. 2018, 47, $32-42$. [CrossRef]

7. Keum, N.-H.; Oh, K.; Hong, S.-K.; Kwon, O.-K. A Pixel Structure Using Block Emission Driving Method for High Image Quality in Active Matrix Organic Light-Emitting Diode Displays. J. Disp. Technol. 2016, 12, 1250-1256. [CrossRef]

8. Fan, C.-L.; Chen, Y.-C.; Yang, C.-C.; Tsai, Y.-K.; Huang, B.-R. Novel LTPS-TFT Pixel Circuit with OLED Luminance Compensation for 3D AMOLED Displays. J. Disp. Technol. 2016, 12, 425-428. [CrossRef]

9. Wu, W.; Zhou, L.; Yao, R.-H.; Peng, J.-B. A New Voltage-Programmed Pixel Circuit for Enhancing the Uniformity of AMOLED Displays. IEEE Electron. Device Lett. 2011, 32, 931-933. [CrossRef]

10. Park, J.; Fujii, M.; Moon, J.; Wang, I. The method to compensate IR-drop of AMOLED display. SID Symp. Dig. Tech. Pap. 2018, 40, 983-985. [CrossRef]

11. In, H.-J.; Kwon, O.-K. A Simple Pixel Structure Using Polycrystalline-Silicon Thin-Film Transistors for High-Resolution Active-Matrix Organic Light-Emitting Diode Displays. IEEE Electron. Device Lett. 2012, 33, 1018-1020. [CrossRef]

12. Liao, C.; Deng, W.; Song, D.; Huang, S.; Deng, L. Mirrored OLED pixel circuit for threshold voltage and mobility compensation with IGZO TFTs. Microelectron. J. 2015, 46, 923-927. [CrossRef]

13. Ashtiani, S.J.; Servati, P.; Striakhilev, D.; Nathan, A. A 3-TFT Current-Programmed Pixel Circuit for AMOLEDs. IEEE Trans. Electron. Devices 2005, 52, 1514-1518. [CrossRef]

14. Chaji, G.; Nathan, A. A Fast Settling Current Driver Based on the CCII for AMOLED Displays. J. Disp. Technol. 2005, 1, 283-288. [CrossRef]

15. In, H.-J.; Oh, K.-H.; Lee, I.; Ryu, D.-H.; Choi, S.-M.; Kim, K.-N.; Kim, H.-D.; Kwon, O.-K. An Advanced External Compensation System for Active Matrix Organic Light-Emitting Diode Displays With Poly-Si Thin-Film Transistor Backplane. IEEE Trans. Electron. Devices 2010, 57, 3012-3019. [CrossRef]

16. Bang, J.-S.; Kim, H.-S.; Kim, K.-D.; Kwon, O.-J.; Shin, C.-S.; Lee, J.; Cho, G.-H. A Hybrid AMOLED Driver IC for Real-Time TFT Nonuniformity Compensation. IEEE J. Solid-state Circuits 2016, 51, 966-978.

17. Lin, C.-C.; Yen, S.; Hsu, S.-Y.; Kuo, T.-W.; Lee, T.-S.; Liu, C.-H.; Lin, S.-L.; Hsiao, H.-Y.; Kung, C.-H.; Yeh, Y.-F.; et al. 7-3: AMOLED Pixel Structure Using the Close Loop Negative Feedback Method for High Resolution Displays. SID Symp. Dig. Tech. Pap. 2016, 47, 61-64. [CrossRef]

18. Lee, J.-P.; Jeon, H.-S.; Moon, D.-S.; Bae, B.S. Threshold Voltage and IR Drop Compensation of an AMOLED Pixel Circuit Without a VDD Line. IEEE Electron. Device Lett. 2013, 35, 72-74. [CrossRef]

19. Lin, C.-L.; Lai, P.-C.; Shih, L.-W.; Hung, C.-C.; Lai, P.-C.; Lin, T.-Y.; Liu, K.-H.; Wang, T.-H. Compensation Pixel Circuit to Improve Image Quality for Mobile AMOLED Displays. IEEE J. Solid-state Circuits 2018, 54, 489-500. [CrossRef]

20. Kim, Y.W.; Kwak, W.K.; Lee, J.Y.; Choi, W.S.; Lee, K.Y.; Kim, S.C.; Yoo, E.J. 9.1: 40 Inch FHD AM-OLED Display with IR Drop Compensation Pixel Circuit. SID Symp. Dig. Tech. Pap. 2009, 40, 85. [CrossRef]

21. Lee, J.; Choi, B. Effects of Channel Type and Doping on Hysteresis in Low-Temperature Poly-Si Thin-Film Transistors. IEEE Trans. Electron. Devices 2018, 65, 986-994. [CrossRef] 
22. Hwang, H.W.; Hong, S.; Hwang, S.S.; Kim, K.W.; Ha, Y.M.; Kim, H.J. Analysis of Recoverable Residual Image Characteristics of Flexible Organic Light-Emitting Diode Displays Using Polyimide Substrates. IEEE Electron. Device Lett. 2019, 40, 1108-1111. [CrossRef]

23. Chen, M.; Lei, J.; Huang, S.; Liao, C.; Deng, L. Poly-Si TFTs integrated gate driver circuit with charge-sharing structure. J. Semicond. 2017, 38, 55001. [CrossRef]

24. Radfar, M.; Shah, K.; Singh, J. A highly sensitive and ultra low-power forward body biasing circuit to overcome severe process, voltage and temperature variations and extreme voltage scaling. Int. J. Circuit Theory Appl. 2013, 43, 233-252. [CrossRef]

25. Anthony, B.; Xavier, A.; Frederic, C.; Dominique, D.; Jean, B.B. A robust inverter-based amplifier versus PVT for discrete-time integrators. Int. J. Circuit Theory Appl. 2018, 46, 2160-2169.

C 2020 by the authors. Licensee MDPI, Basel, Switzerland. This article is an open access article distributed under the terms and conditions of the Creative Commons Attribution (CC BY) license (http://creativecommons.org/licenses/by/4.0/). 